\title{
Women's Health
}

National Cancer Institute

\section{Source}

National Cancer Institute. Women's Health. NCI Thesaurus. Code C17399.

Many diseases and disorders affect men and women differently. Some predominantly effect one or the other while others take different clinical courses, with differing prognoses and outcomes. Women's health takes a specific focus on the health and wellbeing of women. 